\title{
QTCP: an optimized and improved congestion control algorithm of high-speed TCP networks
}

\begin{abstract}
TCP researchers evaluated the performance and fairness of different TCP protocols on the basis of new algorithms. The new High-Speed Transport Control Protocols (HS-TCP) were developed but there are still many problems regarding to bandwidth utilization, throughput and packet loss rate. To overcome these problems Quick Transport Control Protocol (QTCP) algorithm based on optimizations of HS-TCP slow start algorithm and Additive Increase and Multiplicative Decrease (AIMD) algorithm have been proposed. A modified algorithm has been developed by using an additive increase approach to grow window with normal speed and to increase scalability by putting constant value of stability of timeline in congestion avoidance phase. This constant timeline gives long stability time; it provides many benefits as compared to other high-speed TCP protocols. The improved algorithm increased throughput and decreased packet loss rate and fairly share link utilization. In this regards several experiment of simulations were observed the fairness. The results show best bandwidth utilization, improved throughput and less packet loss rate as compared to other high speed TCP variants.
\end{abstract}

Keyword: Fairness; QTCP; AIMD; Congestion avoidance; Throughput 